\title{
NIHSS 0: meta ideal y alcanzada en una paciente joven con EVC isquémico de grandes vasos
}

\author{
NIHSS 0: ideal goal achieved in a young patient with ischemic great vessel CVD
}

\author{
Edwin Mejía-Vaca ${ }^{1 *}$, Enrique Castellanos-Pedroza², Gustavo A. Venegas-Valles², Javier Lagos-Servellón² \\ y Dulce M. Bonifacio-Delgadillo²
}

${ }^{1}$ Servicio de Urgencias de Adultos, Hospital General Dr. Darío Fernández Fierro; ${ }^{2}$ Terapia Endovascular Neurológica, Centro Médico Nacional 20 de Noviembre. Instituto de Seguridad y Servicios Sociales de los Trabajadores del Estado, Ciudad de México, México

\section{Introducción}

El evento vascular cerebral (EVC) es una causa importante de morbimortalidad, así como de discapacidad. Su presencia representa una limitación física en la mayoría de los casos, la cual dependerá de la localización y la extensión del infarto, la comorbilidad del paciente y, muy importantes, el tiempo transcurrido entre la aparición de los síntomas y la aplicación del tratamiento adecuado.

\section{Descripción del caso}

Mujer de 47 años con antecedentes ginecológicos de 3 embarazos (0 parto, 1 cesárea, 2 abortos en el primer trimestre), sin más antecedentes de importancia.

Inicia su padecimiento actual el día 30/10/2021 a las 17:30 horas, al encontrase comiendo y presentar de manera súbita desviación de la comisura labial a la derecha, debilidad del brazo y la pierna izquierdos, y disartria.

- Tiempo síntomas-urgencias: 30 minutos.

- Tiempo de stroke: 30 minutos.

A su ingreso a la unidad de urgencias presenta los siguientes signos vitales: presión arterial 128/61 mmHg, frecuencia cardiaca 90 latidos por minuto, frecuencia respiratoria 18 respiraciones por minuto, temperatura $36{ }^{\circ} \mathrm{C}$, glucosa $89 \mathrm{~g} / \mathrm{dl}$.
- Tiempo ingreso-evaluación: 25 minutos.

- Tiempo de stroke: 55 minutos.

Se realiza evaluación integral de la paciente y se calcula una puntuación en la escala NIHSS (National Institute of Health Stroke Scale) de 7 puntos: parálisis facial 2 puntos, derivación del brazo izquierdo 1 punto, pérdida de sensibilidad 2 puntos, negligencia táctil y auditiva 2 puntos.

Se integra la posibilidad diagnóstica de estar cursando con EVC de tipo isquémico. La unidad tiene problemas técnicos del equipo de tomografía, por lo que se activa la red institucional de atención de infarto cerebral agudo, donde se acepta el traslado a una unidad de tercer nivel. Por cuestiones de disposición de recursos materiales, así como de tráfico dentro de la ciudad, deciden enviar unidad y equipo de traslado para continuar en tiempos del stroke chain of survival. ${ }^{1,2}$ Se logra la reactivación del equipo de tomografía en la unidad y se logra tomar una tomografía simple de cráneo.

- Tiempo evaluación-tomografía: 74 minutos (puerta-tomografía: 209 minutos).

- Tiempo de stroke: 239 minutos.

Se realiza tomografía simple de cráneo, en la que no se evidencia lesión hemorrágica, con cambio hipodenso en la región del núcleo caudado y la ínsula del lado derecho, por lo que se asigna ASPECTS (Alberta Stroke

\section{Correspondencia:}

*Edwin Mejía-Vaca

E-mail: lavacamejia@gmail.com 2604-6520 @ 2021 Sociedad Mexicana de Medicina de Emergencias, AC. Publicado por Permanyer México SA de CV. Este es un artículo open access bajo la licencia CC BY-NC-ND (http://creativecommons.org/licenses/by-nc-nd/4.0/).
Fecha de recepción: 01-12-2021 Disponible en internet: 07-03-2022 Fecha de aceptación: 21-12-2021 Rev Educ Investig Emer. 2022;4(Supl 1):102-104 DOI: 10.24875/REIE.21000245 www.medicinadeemergencias.com 


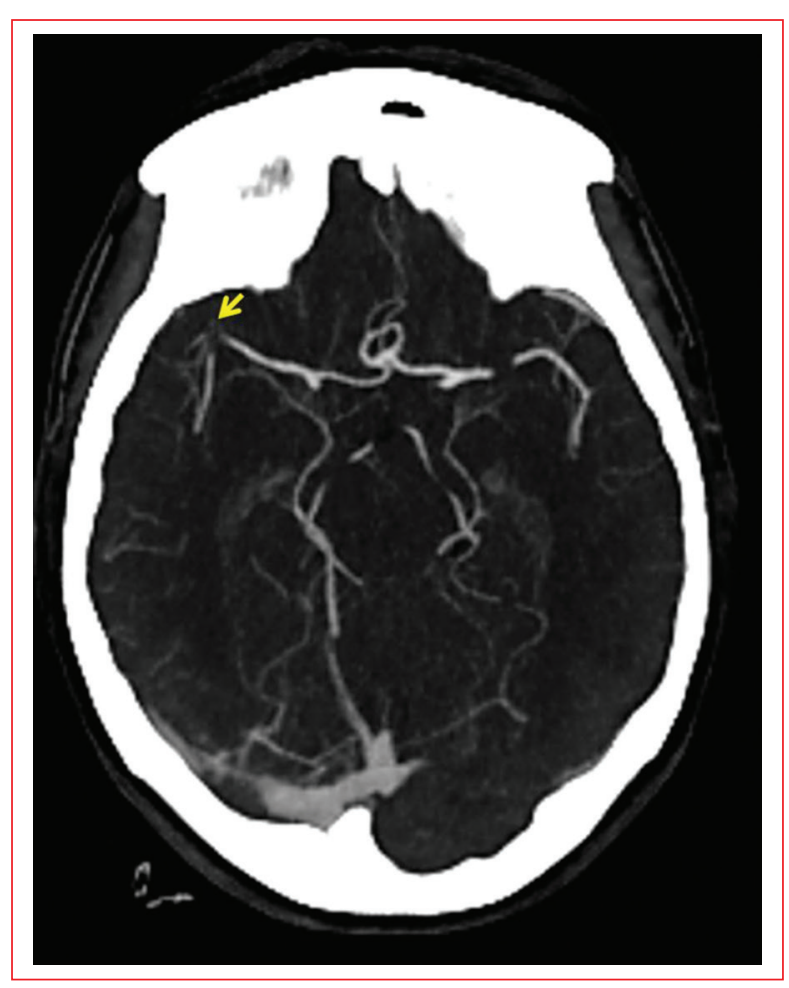

Figura 1. Angiotomografía cerebral (axial, MIP) con oclusión de arteria cerebral media derecha en su segmento M1 distal (flecha).

Programme Early CT Score) 8 puntos y se decide realizar trombólisis previo consentimiento informado?

- Tiempo tomografía-aguja: 10 minutos (puerta-aguja: 249 minutos).

- Tiempo de stroke: 249 minutos.

Se calcula trombolítico, alteplasa, a $0.9 \mathrm{mg} / \mathrm{kg}(48 \mathrm{~kg})$, aplicando en bolo $4.3 \mathrm{ml}$ y dejando en bomba de infusión $38.7 \mathrm{ml}$ para 1 hora. Se traslada al Centro Médico Nacional 20 de Noviembre con bomba de infusión funcional.

- Tiempo traslado-recepción: 19 minutos.

- Tiempo de stroke: 281 minutos.

A su ingreso al Centro Médico Nacional 20 de Noviembre es valorada por el servicio de neurología y terapia endovascular neurológica, quienes en su ingreso reportaron NIHSS de 4 puntos: parálisis facial central leve 1 punto, deriva brazo izquierdo 1 punto, negligencia táctil y auditiva 2 puntos. Se decide realizar imágenes en avanzada para clasificar y delimitar la zona de lesión.

- Tiempo recepción-imagen avanzada: 42 minutos.

- Tiempo de stroke: 357 minutos.

En la angiotomografía se evidencia oclusión de la arteria cerebral media en el segmento M1 distal y se propone realizar angiografía diagnóstico-terapéutica ${ }^{3,4}$ por

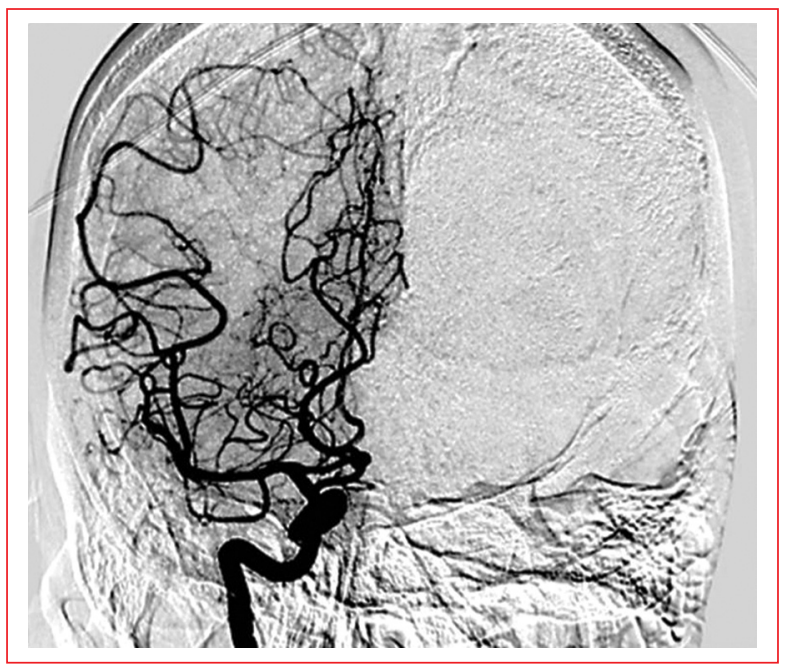

Figura 2. Angiografía cerebral (PA) con recanalización existosa mTICI $2 b$ de ACM derecha después de trombolisis intravenosa con tPA.

el equipo de terapia endovascular neurológica previo consentimiento informado de la paciente y un familiar.

- Tiempo recepción-aguja: 207 minutos.

- Tiempo de stroke: 500 minutos.

- Tiempo puncion - angiografía: 4 minutos.

- Tiempo de stroke: 504 minutos

Hallazgos de la angiografía: recanalización de oclusión sobre la arteria cerebral media derecha; oclusión distal de ramas central y parietal anterior no accesible a terapia de reperfusión endovascular. Pasa a cuidados de post rtPA y a cuidados intensivos.

El día 03/11/2021, a las 13:46 h, se realiza valoración neurológica de la paciente, y en ese momento la puntuación NIHSS es de 0 puntos. Finalmente, la paciente es egresada el día 05/11/2021 con una puntuación en la escala de Rankin modificada de 0.

Al no contar con comorbilidad, de momento no se logró justificar la etiología de su EVC y se consideró indeterminado. Por el breve tiempo del caso, aún no se ha concluido y sigue en protocolos de estudio para determinar las causas.

\section{Perspectiva de la paciente y discusión}

La presentación del EVC isquémico en este caso fue atípico por la edad de la paciente y por no contar con factores de riesgo francos que pudieran desencadenarlo. El traslado oportuno al hospital por parte del familiar fue crucial para lograr tener el tiempo indicado para la aplicación de la trombólisis directa sin necesidad de hacer exámenes de imagen complementarios; si bien fueron necesarios dentro del protocolo, se ganó tiempo 


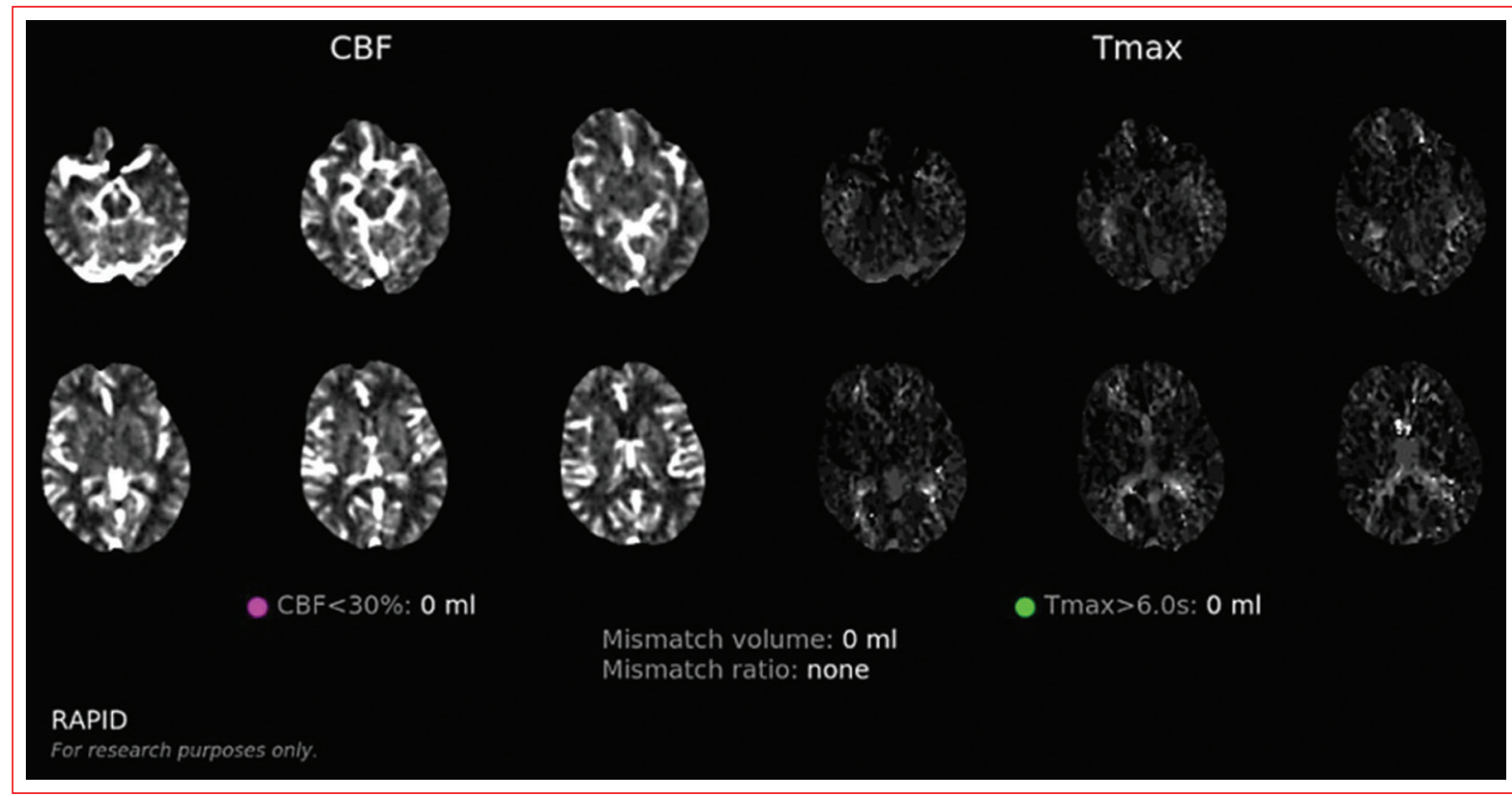

Figura 3. Análisis de perfusión cerebral por tomografía mediante software RAPID -Al que demuestra flujo cerebral normal en territorio de la arteria cerebral media derecha 48 horas después de la terapia de reperfusión.

para la aplicación del medicamento y con ello lograr una recanalización de la circulación de los grandes vasos.

\section{Conclusiones}

EI EVC isquémico representará cada día un problema médico mayor, el cual tendrá una mayor prevalencia en los sistemas de salud, por el envejecimiento de la población y la disminución de la mortalidad, pero gracias a los avances agigantados en los tratamientos médicos y la trombectomía mecánica es posible tratar y con ello reducir la aparición de discapacidad, lo que representa no solo el éxito terapéutico, sino también un ahorro en las arcas públicas de un paciente recurrente y dependiente, y lo más importante, una calidad de vida digna para los pacientes.

\section{Agradecimientos}

Nos sentimos orgullosos y bendecidos al poder participar del programa ResISSSTE cerebro (red interna para el tratamiento del EVC isquémico), en el cual somos testigos y compartimos los éxitos obtenidos logrando mejorar la calidad de vida de los pacientes que han sufrido este tipo de patologías. Y nuestro agradecimiento a todo el personal de limpieza, que dio su mejor esfuerzo e incluso la vida en la pandemia y que tan poco valorado es.

\section{Financiamiento}

Este reporte de caso no cuenta con financiamiento.

\section{Conflicto de intereses}

Este reporte de caso no cuenta con ningún conflicto de intereses.

\section{Responsabilidades éticas}

Protección de personas y animales. Los autores declaran que para esta investigación no se han realizado experimentos en seres humanos ni en animales.

Confidencialidad de los datos. Los autores declaran que han seguido los protocolos de su centro de trabajo sobre la publicación de datos de pacientes.

Derecho a la privacidad y consentimiento informado. Los autores declaran que en este artículo no aparecen datos de pacientes.

\section{Bibliografía}

1. Hacke W, Kaste M, Bluhmki E, Brozman M, Dávalos A, Guidetti D, et al. Thrombolysis with alteplase 3 to 4.5 hours after acute ischemic stroke. N Engl J Med. 2008;359:1317-29.

2. Ma H, Campbell BCV, Parsons MW, Churilov L, Levi CR, Hsu C, et al. Thrombolysis guided by perfusion imaging up to 9 hours after onset of stroke. N Engl J Med. 2019;380:1795-803.

3. Albers GW, Marks MP, Kemp S, Christensen S, Tsai JP, Ortega-Gutiérrez S, et al. Thrombectomy for stroke at 6 to 16 hours with selection by perfusion imaging. N Engl J Med. 2018;378:708-18.

4. Nogueira RG, Jadhav AP, Haussen DC, Bonafe A, Budzik RF, Bhuva P, et al. Thrombectomy 6 to 24 hours after stroke with a mismatch between deficit and infarct. N Engl J Med. 2018;378:11-21. 